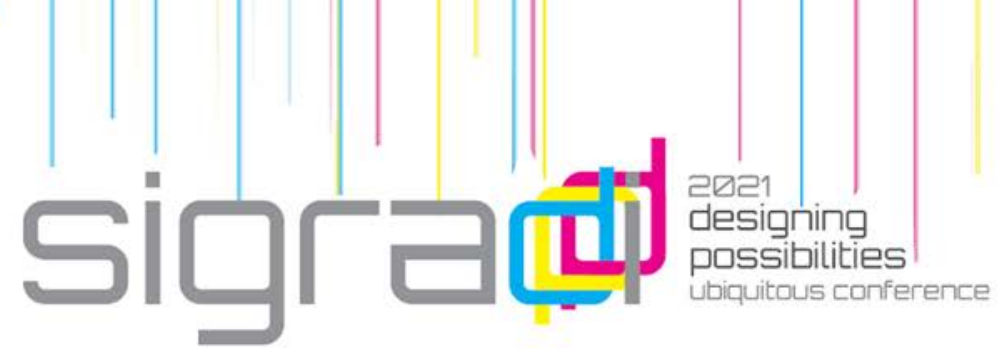

\title{
Design of Self-Organized Structural, Based on the Phenomenon of Crystalline Particle Aggregation
}

\author{
Juan Manuel Villa Carrero, Henry Urley Portilla Delgado \\ University Francisco de Paula Santander, Colombia \\ juanmanuelvc@ufps.edu.co \\ henryurleypd@ufps.edu.co
}

\begin{abstract}
This article deals with the design of structures with the quality of selforganization, applying emergency phenomena in particle aggregation, where a transformation of energy takes place towards structures and states of great complexity from rules of low level of sophistication, when there is an exchange of matter and energy with the outside, and are far from equilibrium. This I+D+i design project focuses on the scientific method, which means observing the phenomenon to answer: What training principles attributable to self-organization underlie the studied phenomenon? and, thus, being able to hypothesize or simulate a model from a Grasshopper environment fed with oversampling methods through Machine Learnig techniques, which allows us to question: How to apply the principles of formation resulting from the emergency phenomenon studied, to the design of self-organized structures? The results conclude that the quality of self-organization of these emerging structural systems is directly proportional to the similarity between their parts, their nesting capacity, the energy levels with which they interact, and their environment, which allows progress in research at scales and open up new future design and manufacturing possibilities.
\end{abstract}

Keywords: Self-Organization, Emergency Phenomenon, Design, Complex Structures, Architecture, Particle Aggregation.

\section{Introduction}

La armonía del mundo se manifiesta en Forma y Número, y el corazón y el alma y toda la poesía de la Filosofía Natural se encarnan en el concepto de belleza matemática. (Thompson, 1992, p. 326). El diseño basado en la naturaleza es parte de la humanidad desde sus orígenes, estos procesos a lo largo de la historia han estado comúnmente ligados al concepto de mimesis o al sentimiento de inspiración, no obstante, con el correr de los tiempos varios científicos del siglo $\mathrm{XX}$, entre los que se encuentran D'arcy $\mathrm{W}$. Thopmson o 
Lancelot Law Whyte fueron más allá, y conectaron el conocimiento a la forma, y advirtieron sobre las analogías superficiales entre la ciencia y el arte. (Whyte, 1951) Así las cosas, no es extraño que actualmente algunos diseñadores conciben la naturaleza como una realidad objetiva y compleja ligada más al concepto de conocimiento científico, que al concepto de imitación o inspiración repentina, esta diferencia, hoy, permite al diseñador representar los objetos externos a él, a partir de su saber desde una realidad abstracta y matemática, en una especie de materialismo abstracto, como plantea (Zizek, 2014), hasta permitir al diseñador reinterpretar la naturaleza, o sintonizarse con ella hasta alterar la materia constitutiva de esa misma realidad.

En esta misma línea, se encuentran precedentes de investigaciones en relación con los objetivos de esta pesquisa, como por ejemplo, Aggregation: DLA Studies, (Louth et al., 2013), que busca el surgimiento de estructuras intrínsecas en las dinámicas de las aguas del Támesis, o estudios sobre principios para la agregación de partículas y su auto ensamblaje (Camassa et al., 2019). Así como también estudios sobre auto ensamblaje, como la representación de moléculas y su modelado en 3D de (Olson, 2012) o la emergencia de la impresión 4D (Tibbits, 2013). Además de proyectos como Sand Engine, (Luijendijk et al., 2019), o Geo Motor, (Carrero \& Cuadros., 2020) y otros que buscan comprender la auto organización en entornos de mayor tamaño como un nuevo modelo de diseño y auto construcción. De por sí, en nuestro caso, estas ideas se tradujeron en el 2016 en una serie de búsquedas reunidas en el proyecto de investigación Experimentación con la Materia y sus Formas iniciado en el 2017 en el grupo de investigación laboratorio de diseño d_lab. Lo que dio lugar a observaciones y simulaciones desarrolladas en el marco del Grown Matter Workshop (GM_W) en el primer semestre del 2019 en la UFPS, y continuaron con la aplicación de sus resultados en los laboratorios de simulación y fabricación de la UFPS en el año 2020.

Por tanto, este trabajo experimenta con la materia y sus formas en su estado natural, buscando comprender, representar, crear y trabajar junto con los sistemas naturales de formación para su aplicación especialmente en la arquitectura, el diseño y en la obtención de estructuras emergentes. Particularmente este articulo versa sobre la comprensión del fenómeno de agregación de concentraciones minerales en ambientes acuosos sobresaturados en proximidad a partículas de semillas, su representación y posterior reinterpretación para diseñar un sistema estructural, con la cualidad de auto organizarse, propia de los fenómenos de emergencia, donde se produce una transformación de la energía hacia estructuras y estados de gran complejidad a partir de reglas de bajo nivel de sofisticación, cuando hay un intercambio de materia y energía con el exterior, es decir, sistemas alejados del equilibrio (Delgado, 2018). Así pues, esta investigación se cuestiona: ¿Qué 
principios de formación subyacen en el fenómeno estudiado? y ¿Cómo aplicar los principios de formación resultado del fenómeno de emergencia estudiado, para el diseño de sistemas estructurales auto organizados?

\section{Metodología}

La presente investigación, del tipo aplicada, permitió desarrollar el actual proyecto de investigación + creación. El cual se desarrolló a partir del método científico soportado en un razonamiento lógico inductivo, que en este caso buscó una respuesta eficiente al problema de diseño de estructuras auto organizadas, basado en la agregación de partículas. Por lo que se inició con el estudio individual del fenómeno en cuestión a través de su observación en forma directa en el laboratorio. Lo que significó la captura, procesamiento y análisis de su data. Y a su vez conllevo, en una segunda etapa, al planteamiento de la hipótesis o principios generales del fenómeno de agregación. Esta hipótesis se materializó a través de un modelo digital y su simulación en Rhinoceros Grasshopper (R-Gh.), que exigió el uso de técnicas de sobre muestreo como el "Gaussian Mixture Model" (GMM), para la obtención de un mayor volumen de data (McLachlan et al., 2014). Esta simulación fue útil experimentalmente para explicar, predecir y aplicar o extrapolar el fenómeno de agregación. Lo que al final apoyó, en una tercera etapa, la creación de estructuras auto organizadas útiles para la arquitectura, dentro de un marco metodológico heurístico, extrapolado del método (Polya, 1957) que no fue otra cosa distinta a un proceso creativo de prueba y error, que determino la probabilidades de auto organización de las piezas de la estructura y su validación, centrados en criterios de energía, espacio, tiempo y similitud y encastre entre sus piezas.

La primera etapa comenzó por la obtención de agregaciones cristalinas a partir de una solución con soluto de alumbre de potasio $\left(\mathrm{KAl}\left(\mathrm{SO}_{4}\right)_{2}-12 \mathrm{H}_{2} \mathrm{O}\right)$ sobresaturada al $70 \%$, que actuó como una fuerza impulsora de la nucleación del soluto al desequilibrarse el sistema por evaporación térmica. La observación de este fenómeno priorizó la captura, procesamiento y análisis de su data de nucleación, sobre el proceso de crecimiento o cristalización. Es decir, destacó un conjunto finito de muestras, que en este caso fue la medición aleatoria de la aparición y agregación de núcleos o partículas del soluto alrededor de perturbaciones de su entorno (hilo, recipiente y líquido). Para lo cual, se realizaron varias pruebas por separado, y cada una de ellas geo referenció el fenómeno en diferentes variables en $\mathrm{X}, \mathrm{Y}$ y $\mathrm{Z}$ a intervalos de tiempo (t) de agregación, lo que constituyo la identificación de la muestra. Estas mediciones se registraron con tres cámaras que grabaron a $60 \mathrm{fps}$, y se procesaron a mano, a través de mapeos raster "frame to frame" o $4 \mathrm{~d}$ en una grilla de $5 \mathrm{~mm} \times 5 \mathrm{~mm}$, cada 5 minutos durante 1 hora, posteriormente estos datos se analizaron, y sistematizaron en tablas Excel. Esta data en Excel, o 


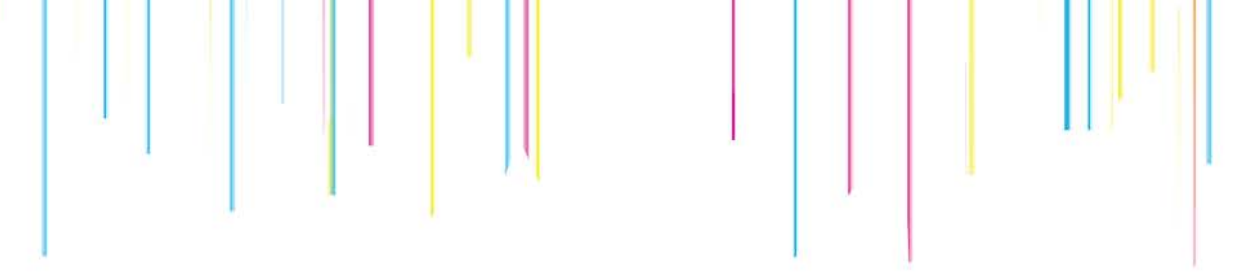

conjunto estadístico de elementos se constituyó en la base del modelo de simulación y del análisis predictivo posterior, que se explicará a continuación. Figura 1

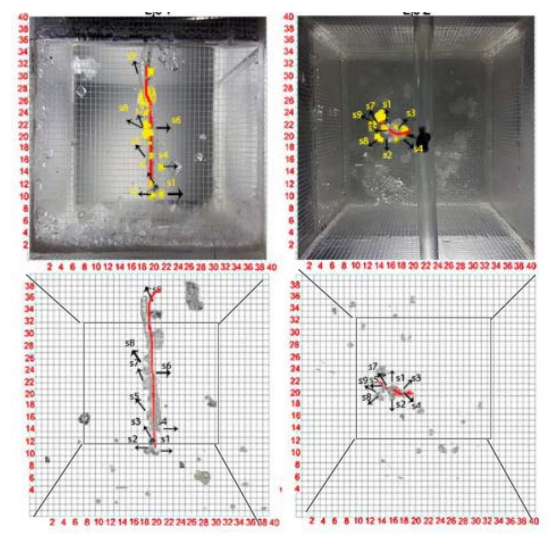

Figure 1. Fragmentos de fotogramas y mapeos del fenómeno de agregación de partículas, captura de data de las variables de tiempo $(\mathrm{t})$ y espacio, $(\mathrm{X}, \mathrm{Y}, \mathrm{y} \mathrm{Z})$. Fuente: GM_W, Mantilla J. y Ortega S., 2019.

En la segunda etapa se transfirió la materia análoga estudiada, tanto en forma de variables en una tabla de Excel, como en un modelo digital. Lo cual se convirtió en una hipótesis del fenómeno de agregación estudiado, abstrayendo y codificando en un editor de programación visual R-Gh. los rasgos más relevantes de su proceso de auto organización: las coordenadas de emergencia de las partículas $\mathrm{X}, \mathrm{Y}, \mathrm{y} Z$ Z, el tiempo (t) de aparición de núcleos, y su área de encastre. Para lo cual se utilizó principalmente dos plugin de RGh, Exread que ayudo a importar las variables de la muestra, y Generation que controló la cantidad y velocidad de agregación, junto con su registro que conservo una nube de puntos. La cual, se logró encastrar entre sí apoyados en variaciones de crecimientos entre caras (Kirdeikis \& Vestartas, 2017). Todo ello dentro de un espacio digital cartesiano en torno a perturbaciones específicas. Esta simplificación del fenómeno significo excluir de la simulación la alteración continuada del resto del entorno a causa del crecimiento o agrupación de partículas aisladas. Y al mismo tiempo implicó utilizar esta data o muestra espacio temporal de aparición de las agregaciones, como la única guía para entender las lógicas de trayectoria del fenómeno. Sin embargo, la visualización del fenómeno con la data inicial desvelo las limitaciones de escala del fenómeno recreado en el laboratorio y la necesidad de obtener un mayor conjunto o muestra de data, de la obtenida originalmente, que ampliará la perspectiva de comprensión del crecimiento de este proceso de agregación dentro de la definición de R-Gh, sin recurrir nuevamente a la experimentación análoga a escalas mayores. Figura 2. 

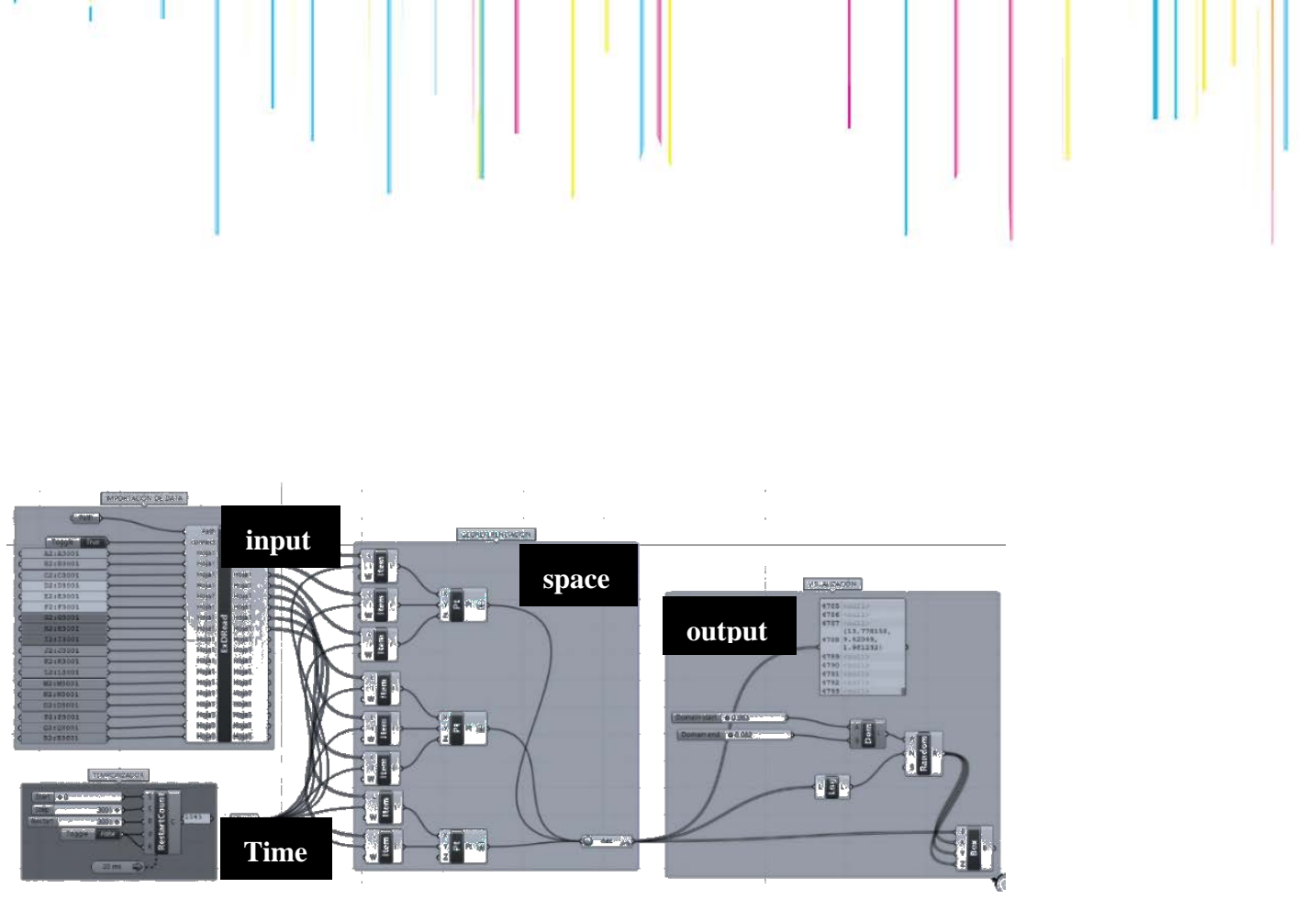

Figure 2. Definición final de la simulación del proceso de agregación de partículas estudiado en un entorno Rhinoceros Grasshopper (R-Gh). Fuente: GM_W, autores, ,2019

Por tanto, se recurrió a proyecciones probabilísticas propias de Excel, pero sin éxito. Lo que obligo posteriormente a extender la data original a partir de métodos predictivos de Machine Learnig que mejoraron el desempeño de la simulación, más exactamente se utilizó un método de sobre muestreo a partir de "Gaussian Mixture Model" (GMM). Este método GMM se realizó, en particular, con la ayuda de Scikit-learn librería más útil para Machine Learning en Python, para no especialistas, desplegado dentro de la plataforma del desarrollador Anaconda. (Pedregosa et al., 2011) Este módulo de Python Scikit-learn permitió el acceso a algoritmos de código abierto dentro de librerías de organización, graficación, análisis y proyección de data como Panda, Matplotlib, Numpy, Seaborn. Que en el caso de esta pesquisa partió de las características o medidas en cada una de las columnas de la tabla de Excel procesada al inicio, en lo que se conoce en el ámbito del aprendizaje estadístico como "features", es decir, las columnas X, Y, Z, y (t) caracterizadas en Excel. Así pues el GMM parametrizó y distribuyó las probabilidades de medidas, con el cual se pudo analizar la estructura intrínseca o inherente del dominio de los datos, (Reynolds, 2009). Y con ello, este método de sobre muestreo GMM aumentó el conjunto de muestras originales de esta investigación creando nuevas pseudo-muestras, que a pesar de no haber existido, contienen la información estadística de las muestras del conjunto original. Lo que permitió en nuestro caso realimentar el modelo del fenómeno de agregación en Grasshopper aumentando la entrada de data, por ejemplo, de un rango entre 30 - 50 factores numéricos a más de 500,1000, 2000, o 3000 factores. Este crecimiento de la información de las variables de la muestra permitió conservar sus lógicas de crecimiento, por tanto comprender el fenómeno en el tiempo y a la vez validar sus resultados comparándolos con las observaciones originales.

La última etapa, se centró en el diseño, para lo cual se utilizó el método de ensayo y error, es decir una técnica heurística que buscó implementar un bucle metodológico que involucró cuatro pasos: entender, planear, ejecutar, revisar. 
Esta estrategia de creación buscó hallar una respuesta de diseño coherente con principios de auto organización: energía, espacio, tiempo, similitud y encastre de sus piezas, ya caracterizados en las dos primeras etapas. Esta etapa inició con la experimentación con piezas existentes con capacidad de encastre frente a entornos dinámicos, para luego, re diseñar piezas propias mejoradas, también expuestas a las misma condiciones, para lo cual, contamos con técnicas de fabricación "3d Fused Deposition Modeling", con una impresora MakerBot con filamento en PLA y PLA touch.

\section{Resultados}

Los primeros resultados respondieron a la siguiente cuestión ¿Qué principios de auto organización subyacen en el fenómeno estudiado? los resultados explicaron que este fenómeno de auto organización se relacionara tanto con los niveles energéticos con los que interactuó, como con su entorno, la similitud entre sus partes, y su capacidad de encastre, lo cual favoreció la formación de estructuras nuevas. Puntualmente, en este fenómeno de emergencia las partículas surgieron y se auto organizaron impulsadas en medio de una fuerza hidrodinámica (movimiento Browniano) y reglas de bajo nivel de sofisticación. Que llevó estas partículas hacia estructuras y estados de gran complejidad, cuando hay un intercambio de materia y energía con el exterior, es decir, sistemas alejados del equilibrio, (Delgado, 2018). Asimismo se apreció cambios de dirección en el proceso de agregación. Esto ocurrió cada vez que el desarrollo de auto organización fue perturbado, ya fuese por su entorno de surgimiento o por la alteración continuada de este por el crecimiento o agrupación de partículas. Sin embargo, la agregación de partículas en torno a estas perturbaciones, como las agregaciones libres de estas, tendió siempre hacia órdenes o Acreciones Simétricas Omnidireccionales (ASO) como lo confirmó los modelos matemáticos de principios para la agregación de partículas y el auto ensamblaje en fluidos estratificados elaborados por (Camassa et al., 2019). Así como también, lo confirman los resultados de nuestras simulaciones en R-Gh con la data del GMM.

Esto ocurrió tanto en las trayectorias advertidas con respecto a una línea, un solo plano (superficie), o cuando se generaron protuberancias, o se hicieron hendiduras sobre un plano. Por ejemplo, en el primer caso se observó que las agregaciones tendieron a un elipsoide $\mathrm{o}$ un esferoide prolato, otras veces oblato, si vemos la línea o hilo sembrado como un eje de simetría. Este elipsoide mantuvo su dirección y escala mientras se conservó la homogeneidad del entorno de auto organización. En el segundo caso, se formaron discos con los núcleos que emergieron en la superficie. Figura 3. 


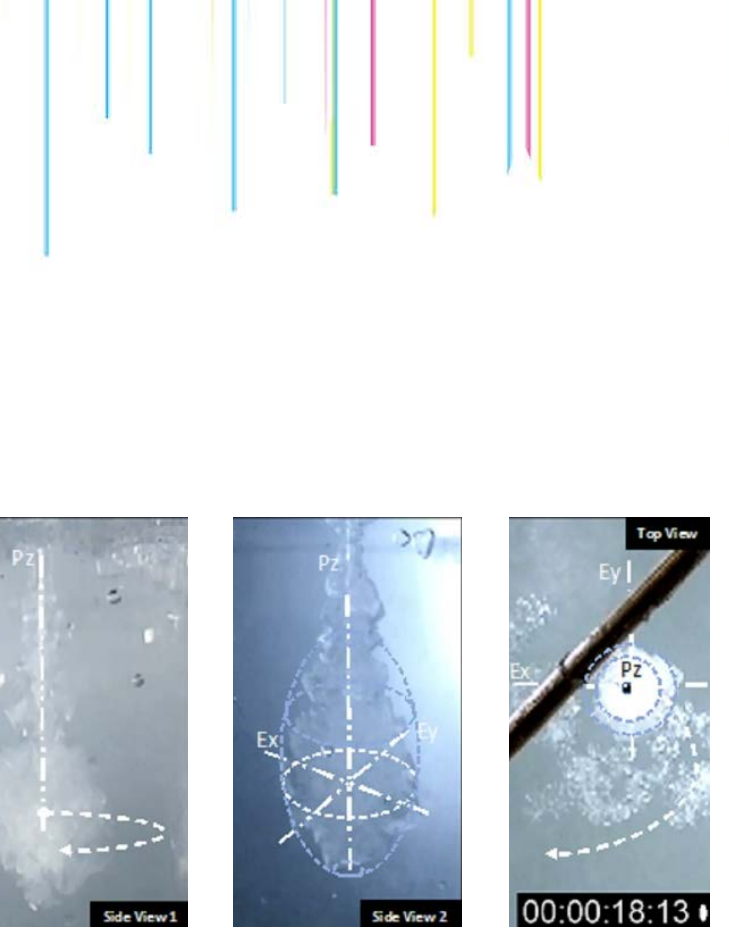

Figure 3. Análisis de los autores sobre fotograma acreción elipsoidal, vistas laterales, y en forma de un gran disco en la superficie, vista superior. Fuente: GM_W, Conde J. y Quintero M, 2019.

Y en un tercer caso se labró una retícula sobre un plano, y como se esperaba las partículas se agregaron a lado y lado de sus líneas, como también ocurrió en las pesquisas de (Louth et al., 2013). De hecho, en nuestro caso, al intersectarse las agregaciones propendieron a inscribir círculos dentro de los cuadrados de la retícula. Figura 4.
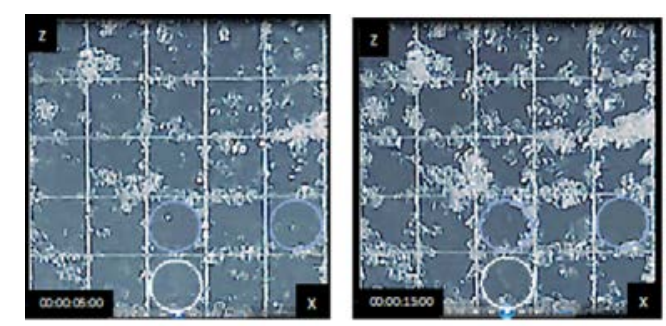

Figure 4. Análisis de los autores sobre fotograma agregación de partículas de perturbaciones en un plano. Fuente: GM_W Conde J. y Quintero M., 2019.

Los resultados de las pesquisas anteriores, si se recuerda, sirvieron como base de un modelo del fenómeno representado a través de una simulación, que visualizó la data en un entorno R-Gh, esta simulación sistematizó tres aspectos básicos del fenómeno estudiado: el tiempo (t) que definió la dinámica provocada por el desequilibrio energético, el espacio $(x, y, z)$ que geo referenció la agregación activada por la sobresaturación del soluto y perturbaciones en el entorno, y el maclado de las piezas, que definió el área de encastre entre 1/4 y $2 / 4$ de la superficie total de la cara de las piezas. Los resultados de estas pruebas digitales, producto de pseudo muestras, fueron contrastadas con las pruebas análogas con el soluto durante la primera etapa o fase de observación del fenómeno estudiado. Esta comparación pudo constatar que las pruebas digitales se aproximaron razonablemente a las pruebas análogas iniciales, entre un $60 \%$ a $80 \%$. Lo que validó tanto estas pseudo-muestras como la definición en R-Gh, base de la simulación utilizada para este trabajo. Figura 5 

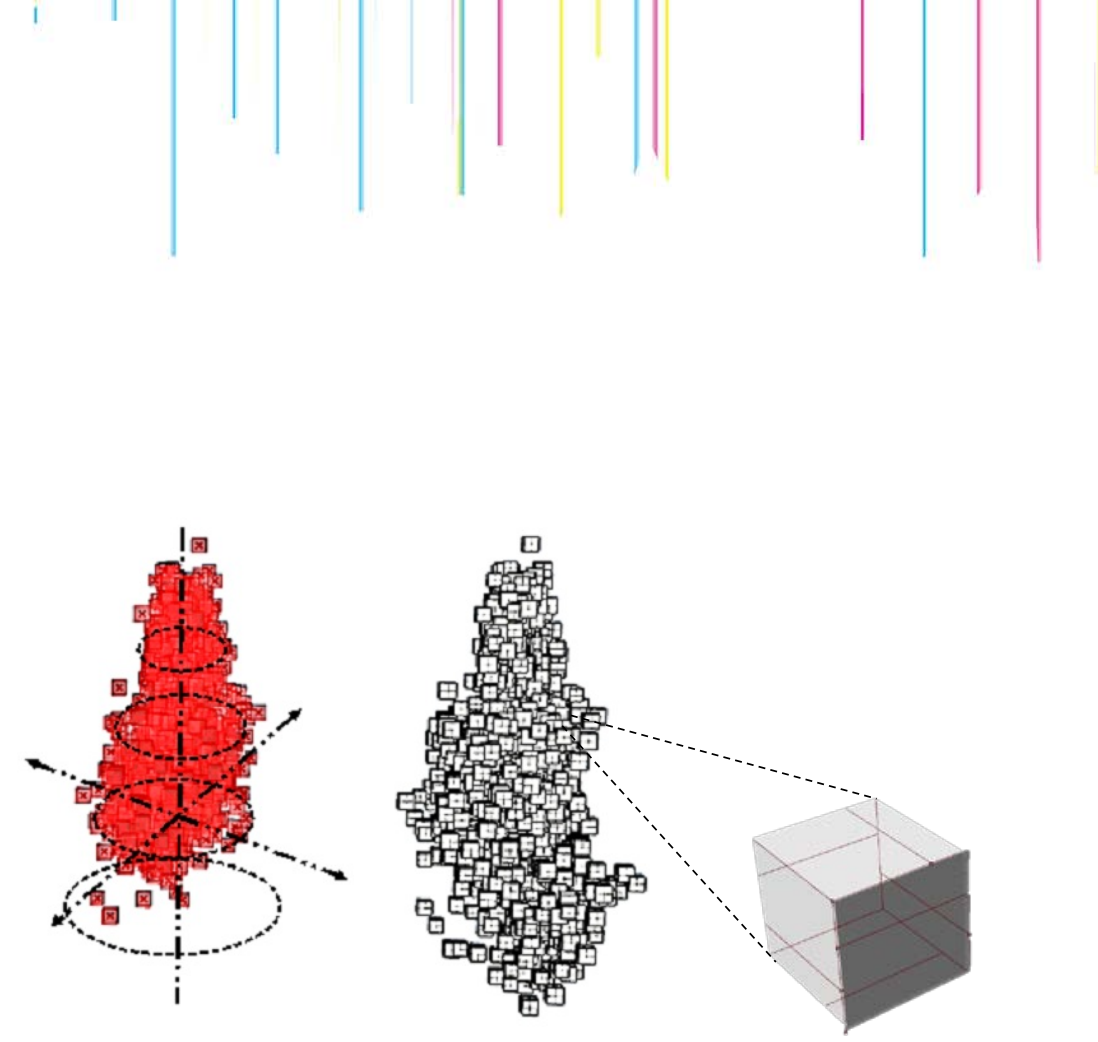

Figure 5. Imágenes simulación de agregación de partículas y detalle de su área de encastre (1/4 a 2/4) en Rhinosceros Grasshopper. Fuente: Autores ,2020.

Por otro lado, una vez validada la etapa de observación y simulación, se respondió a la cuestión: ¿Cómo aplicar los principios de formación resultado del fenómeno de emergencia estudiado, para el diseño de estructuras auto organizadas? La respuesta a esta pregunta se canalizó a través de una estrategia heurística, en otras palabras, primero habiendo entendido los resultados anteriores, se demostró, en segundo lugar, la necesidad de establecer un plan que captara la relación sistémica entre las partes del fenómeno; energía (t), entorno ( $x, y, z)$, y pieza. Por lo que, en tercer lugar, al igual que los trabajos de (Olson, 2012) y (Tibbits, 2013), se experimentó con la aplicación de energía a un grupo de piezas, en este caso, del popular juego denominado Jacks. Estas piezas de juego mostraron como se esperaba un alto potencial de encastre, que dentro de un entorno definido (recipiente) al interactuar provocaron la auto organización entre ellas, el resultado de esta dinámica generó una estructura más compleja, pero al someterse a una revisión mostró ser muy inestable. Figura 6.
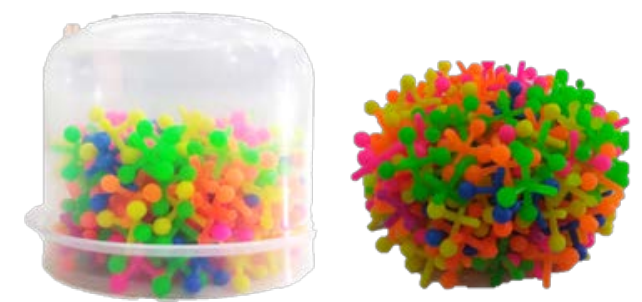

Figure 6. Experimento 1, juego de Jacks, pieza abierta, Fuente: Autores 2020

Así pues, esta revisión de los resultados desvelo la inestabilidad de la estructura entre las piezas del juego de Jacks, lo que direccionó el segundo ensayo y bucle de pruebas, el cual consideró el diseño y fabricación de nuevas piezas que no solamente permitieran la auto organización como el anterior 


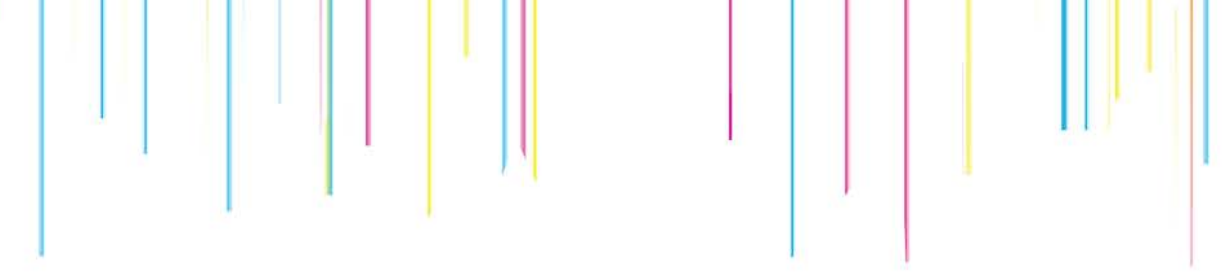

experimento, si no, que también, almacenaran la energía una vez ocurriera el encastre entre ellas, es decir, se buscó que sus piezas conservaran la estabilidad estructural. En este orden de ideas, se diseñó una pieza cerrada, a diferencia de la pieza de Jacks, en este caso se optó por un tetraedro perforado en tres de sus vectores y abultado en sus nodos, esta pieza se fabricó y repitió varias veces en PLA y se expusieron a un movimiento continuo (energía) en un recipiente durante seis minutos, los resultados de estas pruebas permitieron auto organizar dos tipos de estructuras: una en forma de cadena, que conservó su enlace una vez cesó el movimiento, y otra unida entre sí, pero inestable por el abultamiento de sus nodos, lo fino de sus vectores y la debilidad de su material. Figura 7.

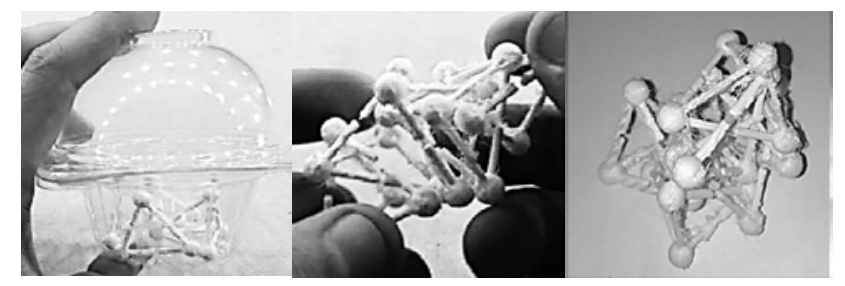

Figure 7. Experimento 2, prototipo de pieza cerrada PLA, Fuente: Autores, 2020.

Por último, se solventaron los errores del ensayo anterior, en otras palabras, se optimizo la pieza sustituyendo el material por uno más resistente, PLA Touch, se conservó la pieza cerrada, pero se engrosaron sus vectores, se redujo a dos sus perforaciones, y se eliminó el abultamiento en sus nodos, y se repitió el segundo ensayo, durante los mismos seis minutos con todos estos cambios. Los resultados de esta nueva versión de la pieza probó la eficiencia formal de esta y un comportamiento estructural más estable, tanto en la estructura auto organizada en cadena, como en su versión compacta. Figura 8.
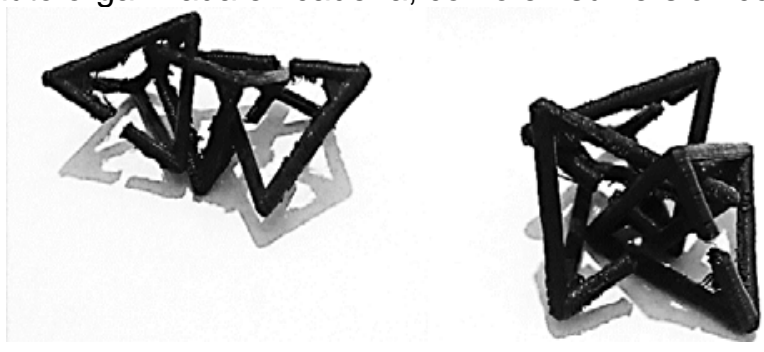

Figure 8. Experimento 3, pieza cerrada PLA Touch, estructura en cadena y compacta, Fuente: Autores, 2020.

En conclusión, para el diseño de estructuras auto organizadas, basadas en el fenómeno de emergencia que ocurre en concentraciones minerales en ambientes acuosos sobresaturados en proximidad a partículas de semillas, se necesitó tener en cuenta tres principios básicos: energía, una circunscripción espacial determinada o entorno y un conjunto de piezas con potencial de 
encastre. Así mismo, según esta investigación se determinó que la forma del entorno y las piezas proveyeron la dirección de crecimiento o formación de estas estructuras auto organizadas. Lo que prueba que estos resultados en teoría, pueden ser extrapolados a escalas mayores como las alcanzadas en trabajos como Sand Engine, (Luijendijk et al., 2019), que logra con partículas de arena conservar la extensión de playa ganada al mar en Holanda, o Geo Motor, (Carrero et al.,2020) que propone direccionar sedimentos rocosos en ríos de montaña.

\section{Discusión}

Para comenzar la discusión es importante recapitular y recordar que este proyecto investiga, desarrolla e innova estructuras que pueden auto organizarse. Los resultados de estas estructuras, generalizando, se ajustan a las pruebas realizadas y mapeadas con el soluto durante el GM_W, tanto como con el modelo simulado digitalmente del fenómeno estudiado, lo que los valida, y a su vez permite compararlos con los resultados alcanzados por autores como (Olson, 2012) y (Tibbits, 2013), que experimentan con el auto ensamblaje. Sin embargo, esta pesquisa a diferencia de estos autores no busca ensamblar estructuras prediseñadas, si no busca comprender las reglas de auto organización, para luego direccionarlas, y por ende, ser capaz de diseñar estas estructuras de emergencia, lo que significa que se busca explorar la posibilidad de proyectar y fabricar simultáneamente como se infiere en los resultados expuestos en este artículo. Lo que sitúa esta investigación más en la línea de proyectos como Sand Engine, (Luijendijk et al., 2019), Geo Motor, (Carrero \& Cuadros., 2020) y otros que buscan comprender la auto organización como un nuevo modelo de diseño y fabricación. Como también es el caso de Aggregation: DLA Studies (Louth et al., 2013), quienes, apoyados en fenómenos similares a los de esta pesquisa, observaron una acreción omnidireccional en la agregación de concentraciones minerales a partículas de semilla, como esta pesquisa. No obstante, los resultados de nuestra investigación discrepan de los de los modelos de trayectorias de agregaciones simulados por este último proyecto. Que a diferencia de los nuestros, al simular, no obtuvieron trayectorias elipsoidales de agregación equivalentes a las pruebas con el material, sino trayectorias lineales continuas y homogéneas.

Por otro lado, aunque se logró modelar el fenómeno y simular escalas mayores digitalmente, el proyecto afrontó problemas al escalar de forma análoga las estructuras auto organizadas en entornos superiores a los experimentados, tanto por el espacio, los niveles de energía, como por el volumen de piezas necesario para esta empresa, por cierto, este proyecto aún no mide los niveles de temperatura en relación con el maclado de las piezas, lo que ayudaría a su conexión como sugiere (Olson, 2012), sin embargo, estas limitaciones son oportunidades para continuar con otras investigaciones donde 
